PROCEEDINGS OF THE

AMERICAN MATHEMATICAL SOCIETY

Volume 131, Number 5, Pages 1527-1538

S 0002-9939(02)06703-5

Article electronically published on September 19, 2002

\title{
TIGHT FRAME OVERSAMPLING AND ITS EQUIVALENCE TO SHIFT-INVARIANCE OF AFFINE FRAME OPERATORS
}

\author{
CHARLES K. CHUI AND QIYU SUN
}

(Communicated by David R. Larson)

\begin{abstract}
Let $\Psi=\left\{\psi_{1}, \ldots, \psi_{L}\right\} \subset L^{2}:=L^{2}(-\infty, \infty)$ generate a tight affine frame with dilation factor $M$, where $2 \leq M \in \mathbf{Z}$, and sampling constant $b=1$ (for the zeroth scale level). Then for $1 \leq N \in \mathbf{Z}, N \times$ oversampling (or oversampling by $N$ ) means replacing the sampling constant 1 by $1 / N$. The Second Oversampling Theorem asserts that $N \times$ oversampling of the given tight affine frame generated by $\Psi$ preserves a tight affine frame, provided that $N=N_{0}$ is relatively prime to $M$ (i.e., $\operatorname{gcd}\left(N_{0}, M\right)=1$ ). In this paper, we discuss the preservation of tightness in $m N_{0} \times$ oversampling, where $1 \leq m \mid M$ (i.e., $1 \leq m \leq M$ and $\operatorname{gcd}(m, M)=m$ ). We also show that tight affine frame preservation in $m N_{0} \times$ oversampling is equivalent to the property of shiftinvariance with respect to $\frac{1}{m N_{0}} \mathbf{Z}$ of the affine frame operator $Q_{0, N_{0}}$ defined on the zeroth scale level.
\end{abstract}

\section{INTRODUCTION AND RESULTS}

A family $\Psi=\left\{\psi_{1}, \ldots, \psi_{L}\right\} \subset L^{2}:=L^{2}(-\infty, \infty)$ is said to generate a tight affine frame

$$
\mathcal{F}_{1}=\left\{\psi_{l ; j, k}(x):=M^{j / 2} \psi_{l}\left(M^{j} x-k\right): j, k \in \mathbf{Z}, l=1, \ldots, L\right\}
$$

of $L^{2}$ with dilation factor $M$ where $2 \leq M \in \mathbf{Z}$ (or for simplicity, we say that $\Psi$ is a tight affine frame of $L^{2}$ ), if there exists a positive constant $A$, called frame (bound) constant, such that

$$
\sum_{l=1}^{L} \sum_{j, k \in \mathbf{Z}}\left|\left\langle f, \psi_{l ; j, k}\right\rangle\right|^{2}=A\|f\|_{2}^{2} \quad \text { for } \quad \text { all } f \in L^{2} .
$$

Here, the standard notation for $L^{2}$-inner product and $L^{2}$-norm is used. In addition, the definition

$$
\hat{f}(\omega):=\int_{-\infty}^{\infty} f(x) e^{-i x \omega} d x, f \in L^{1},
$$

Received by the editors February 8, 2001 and, in revised form, December 16, 2001

2000 Mathematics Subject Classification. Primary 42C40.

The research of the first author was partially supported by NSF Grant \#CCR-9988289 and ARO Grant \#DAAD 19-00-1-0512.

The second author is also a visiting member of the Institute of Computational Harmonic Analysis, University of Missouri-St. Louis. 
of the Fourier transform will be used throughout this paper. Also, for any $\Psi=$ $\left\{\psi_{1}, \ldots, \psi_{L}\right\} \subset L^{2}$ that satisfies the property

$$
H(\omega):=\sum_{l=1}^{L} \sum_{k \in \mathbf{Z}}\left|\widehat{\psi}_{l}(\cdot+2 k \pi)\right|^{2} \in L^{\infty}(-\infty, \infty),
$$

we consider the affine frame operators $Q_{0, n}, 1 \leq n \in \mathbf{Z}$, defined by

$$
\left(Q_{0, n} f\right)(x):=\sum_{l=1}^{L} \sum_{k \in \mathbf{Z}}\left\langle f, \psi_{l}(\cdot-k / n)\right\rangle \psi_{l}(x-k / n), \quad f \in L^{2},
$$

on the zeroth scale level, and denote $Q_{0}:=Q_{0,1}$. Clearly, we have

$$
\left\|Q_{0, n} f\right\|_{2} \leq n\|H\|_{\infty}\|f\|_{2} .
$$

The reason for the terminology of "affine frame operators" is that, by introducing the dilation operator

$$
D_{j} f:=f\left(M^{j} \cdot\right), \quad j \in \mathbf{Z},
$$

$\Psi \subset L^{2}$ is a tight affine frame of $L^{2}$ (in the sense that the family $\mathcal{F}_{1}$ in (1) is a tight frame of $L^{2}$ ), if and only if both (3) and, for some constant $A>0$,

$$
\sum_{j \in \mathbf{Z}} D_{-j} Q_{0} D_{j}=A I
$$

are satisfied.

For $M=2$, a complete characterization of tight affine frames (more precisely, orthonormal wavelets) is discussed in 12, and a generalization from $M=2$ to arbitrary real dilation $a>1$ is given in [10]. Generalizations to matrix dilation have been studied in 3, 4, 5] for matrices with integer entries, and most recently in 6] for arbitrary real matrices. Of course, all the eigenvalues of the dilation matrices must have magnitudes greater than one. For the univariate setting with dilation factor $a>1$, where $a^{\gamma}=: n_{a} \in \mathbf{Z}$ for some $1 \leq \gamma \in \mathbf{Z}$, and $\gamma$ being the smallest such integer exponent, the full characterization in [10] reduces to the following.

Theorem A. Let $\Psi=\left\{\psi_{1}, \ldots, \psi_{L}\right\} \subset L^{2}, b \neq 0$ and $a>1$ with

$$
\left\{\begin{array}{l}
a^{\gamma}=: n_{a} \in \mathbf{Z} ; \\
a^{j} \notin \mathbf{Z} \text { for } \gamma>j \in \mathbf{Z} \backslash\{0\} .
\end{array}\right.
$$

Then $\left\{a^{j / 2} \psi_{l}\left(a^{j} \cdot-k b\right): j, k \in \mathbf{Z}, l=1, \ldots, L\right\}$ is a tight frame of $L^{2}$ with frame constant $A$, if and only if

$$
\sum_{l=1}^{L} \sum_{j \in \mathbf{Z}}\left|\widehat{\psi}_{l}\left(a^{j} \omega\right)\right|^{2}=A \quad \text { a.e. } \omega \in \mathbf{R}
$$

and

$$
\sum_{l=1}^{L} \sum_{j=0}^{\infty} \overline{\widehat{\psi}_{l}\left(n_{a}^{j} \omega\right)} \widehat{\psi}_{l}\left(n_{a}^{j}\left(\omega+2 b^{-1} d \pi\right)\right)=0 \quad \text { a.e. } \omega \in \mathbf{R}
$$

for any $d \in \mathbf{Z} \backslash n_{a} \mathbf{Z}$.

Returning to the special case $a=M \in \mathbf{Z}$ and $b=1$, let us also recall the following Second Oversampling Theorem established in [7]. 
Theorem B. Let $2 \leq M \in \mathbf{Z}$ and assume that $\Psi=\left\{\psi_{1}, \ldots, \psi_{L}\right\} \subset L^{2}$ generates a tight affine frame $\mathcal{F}_{1}$ in (11) of $L^{2}$ with frame constant $A>0$. Then for any $2 \leq m \in \mathbf{Z}$ with $\operatorname{gcd}(m, M)=1$,

$$
\mathcal{F}_{m}=\left\{M^{j / 2} \psi_{l}\left(M^{j} x-k / m\right): j, k \in \mathbf{Z}, l=1, \ldots, L\right\}
$$

is also a tight frame of $L^{2}$, with frame constant $m A$.

The notion of oversampling affine frames was first introduced in [8], where the result for $M=2$ was obtained, although [8] has a later publication date than [7], which deals with the theory of affine frames in general. Generalizations to matrix dilation was studied in [9] and, in full generality, in [6]. In addition, oversampling by $M^{k}$ was discussed in [11] for dilation $M=2$ for tight affine frames, and arbitrary $2 \leq M \in \mathbf{Z}$ in [13, Chapter 5] for bi-orthogonal wavelets, where $0<k \in \mathbf{Z}$. A generalization of [11] to matrix dilation was also mentioned in [6]. Observe, however, that since the assumption $\operatorname{gcd}(m, M)=1$ is violated for $m=M^{k}, 0<k \in \mathbf{Z}$, it is necessary to derive additional characterization equations, besides those in (10) for $1<a=n_{a}=M \in \mathbf{Z}$ and $b=1$. For instance, by reducing the matrix consideration in [6] to the scalar setting, a necessary and sufficient condition for tight affine frame preservation in $M^{n_{0}} \times$ oversampling, with $0<n_{0} \in \mathbf{Z}$, is that

$$
\sum_{l=1}^{L} \sum_{j=0}^{n_{0}-1} \overline{\widehat{\psi}_{l}\left(M^{j} \omega\right)} \widehat{\psi}_{l}\left(M^{j}(\omega+2 d \pi)\right)=0 \quad \text { a.e. } \omega \in \mathbf{R}
$$

for all $d \in \mathbf{Z} \backslash M \mathbf{Z}$.

The objective of this paper is to establish necessary and sufficient conditions for tight affine frame preservation in oversampling by $m N_{0}$, where $\operatorname{gcd}\left(N_{0}, M\right)=1$, $1 \leq m \leq M$, and $\operatorname{gcd}(m, M)=m$. One of the equivalent conditions, stated in Theorem 2, is that the affine frame operator $Q_{0, N_{0}}$ in (4), with $n=N_{0}$, is shiftinvariant with respect to $\frac{1}{m N_{0}} \mathbf{Z}$. To facilitate the statement of our results, we need the notation of the shift operator

$$
\tau_{y}: f \longmapsto f(\cdot-y), y \in \mathbf{R} .
$$

Theorem 1. Let $2 \leq M \in \mathbf{Z}$ and assume that $\Psi=\left\{\psi_{1}, \ldots, \psi_{L}\right\} \subset L^{2}$ generates a tight affine frame $\mathcal{F}_{1}$ in (1) of $L^{2}$ with frame constant $A>0$. Consider $2 \leq m \leq M$ with $\operatorname{gcd}(m, M)=m$. Then the following statements are equivalent:

(i) $\mathcal{F}_{m}$ in (11) is a tight frame of $L^{2}$;

(ii) $\sum_{l=1}^{L} \overline{\widehat{\psi}_{l}(\omega)} \widehat{\psi}_{l}(\omega+2 \pi d)=0$ a.e. $\omega \in \mathbf{R}$, for all $d \in \mathbf{Z} \backslash m \mathbf{Z}$;

(iii) $Q_{0}$ is shift-invariant with respect to $\frac{1}{m} \mathbf{Z}$, i.e.,

$$
\tau_{k / m} Q_{0}=Q_{0} \tau_{k / m} \quad \text { for all } k \in \mathbf{Z}
$$

(iv) $Q_{0, m}=m Q_{0}$;

(v) there exists some $2 \pi$-periodic unitary matrix $A(\omega)$ of dimension $L$ such that

$$
e^{-i \omega / m} \widehat{\Phi}(\omega)=A(\omega) \widehat{\Phi}(\omega) \quad \text { a.e. } \omega \in \mathbf{R},
$$

where $\Phi=\left[\begin{array}{lll}\psi_{1} & \ldots & \psi_{L}\end{array}\right]^{T}$ is the column vector of the functions in $\Psi$.

The $2 \pi$-periodic matrix $A(\omega)$ in (15) of Theorem 1 acts like a bases transform on the shift-invariant space generated by $\psi_{l}(\cdot-k), l=1, \ldots, L, k \in \mathbf{Z}$. Such an invertible transform of frames was discussed in [1,13]. In particular, the implication of $(\mathrm{v}) \Longrightarrow(\mathrm{i})$ was given in [13, Theorem 6.1]. 
In addition, the equivalence of (ii) and (v) was proved in 14 for the special case where $L=1, M=2$, and that $\mathcal{F}_{1}$ is an orthonormal basis of $L^{2}$. But the proof in 14] does not seem to have a simple generalization to the study of frames for $L \geq 1$ and $M \geq 2$. In this regard, our proof of (ii) $\Longrightarrow(\mathrm{v})$ is fairly technical.

From (iii) of Theorem 1, we see that the range $W_{0}:=Q_{0} L^{2}$ of the operator $Q_{0}$ is shift-invariant over $\frac{1}{m} \mathbf{Z}$. If $\mathcal{F}_{1}$ in (1) is an orthonormal basis of $L^{2}$, then as in [14, $W_{0}$ is generated by $\ell^{2}$ linear combinations of integer shifts of $\psi_{1}, \ldots, \psi_{L}$; namely,

$$
W_{0}=\left\{\sum_{l=1}^{L} \sum_{j \in \mathbf{Z}} d_{l}(j) \psi_{l}(\cdot-j):\left\{d_{l}(j)\right\}_{j \in \mathbf{Z}} \in \ell^{2}\right\}
$$

and thus the affine frame operator $Q_{0}$ on the zeroth scale level is the projection operator on $W_{0}$, i.e., $Q_{0}^{2}=Q_{0}$. The interested reader is referred to 2 for a study of closedness of the space generated by $\ell^{p}$ linear combinations, $1 \leq p \leq \infty$, of integer shifts in general.

For functions $\Psi=\left\{\psi_{1}, \ldots, \psi_{L}\right\} \subset L^{2}$ and $b \neq 0$, it is easy to check that $\mathcal{F}_{b^{-1}}=\left\{M^{j / 2} \psi_{l}\left(M^{j} \cdot-k b\right): j, k \in \mathbf{Z}, l=1, \ldots, L\right\}$ is a tight frame of $L^{2}$ if and only if $\left\{M^{j / 2} \psi_{l, b}\left(M^{j} \cdot-k\right): j, k \in \mathbf{Z}, l=1, \ldots, L\right\}$ is a tight frame of $L^{2}$, where $\psi_{l, b}=b^{1 / 2} \psi_{l}(b \cdot)$. Let $N_{0}$ be a positive integer with $\operatorname{gcd}\left(N_{0}, M\right)=1$. Then for any tight affine frame $\Psi=\left\{\psi_{1}, \ldots, \psi_{L}\right\}$ of $L^{2}$, it follows from Theorem B that $\left\{M^{j / 2} \psi_{l, N_{0}^{-1}}\left(M^{j} \cdot-k\right): j, k \in \mathbf{Z}, l=1, \ldots, L\right\}$ is also a tight frame of $L^{2}$. Therefore this, together with Theorem 1, give the following extension of Theorem 1.

Theorem 2. Let $2 \leq M \in \mathbf{Z}$ and assume that $\Psi=\left\{\psi_{1}, \ldots, \psi_{L}\right\} \subset L^{2}$ generates a tight affine frame $\mathcal{F}_{1}$ in (1) of $L^{2}$ with frame constant $A>0$. Consider $1 \leq N_{0} \in \mathbf{Z}$ with $\operatorname{gcd}\left(N_{0}, M\right)=1$, and $2 \leq m \leq M$ with $\operatorname{gcd}(m, M)=m$. Then the following statements are equivalent:

(i) $\mathcal{F}_{m N_{0}}$ in (11) is a tight frame of $L^{2}$;

(ii) $\sum_{l=1}^{L} \widehat{\widehat{\psi}_{l}(\omega)} \widehat{\psi}_{l}\left(\omega+2 d N_{0} \pi\right)=0$ a.e. $\omega \in \mathbf{R}$, for all $d \in \mathbf{Z} \backslash m \mathbf{Z}$;

(iii) the operator $Q_{0, N_{0}}$ in (4), with $n=N_{0}$, is shift-invariant with respect to $\left(m N_{0}\right)^{-1} \mathbf{Z}$, i.e.,

$$
\tau_{k /\left(m N_{0}\right)} Q_{0, N_{0}}=Q_{0, N_{0}} \tau_{k /\left(m N_{0}\right)} \quad \text { for all } k \in \mathbf{Z}
$$

(iv) $Q_{0, m N_{0}}=m Q_{0, N_{0}}$;

(v) there exists some $2 \pi$-periodic unitary matrix $A(\omega)$ of dimension $L$ such that

$$
e^{-i \omega /\left(m N_{0}\right)} \widehat{\Phi}(\omega)=A\left(\omega / N_{0}\right) \widehat{\Phi}(\omega) \quad \text { a.e. } \omega \in \mathbf{R}
$$

where $\Phi=\left[\begin{array}{lll}\psi_{1} & \ldots & \psi_{L}\end{array}\right]^{T}$ is the column vector of the functions in $\Psi$.

By applying (7) and (iii) of Theorem 2, we also have the following result for tightness of shifted affine frames.

Corollary 1. Let $M, m$ and $N_{0}$ be as in Theorem 2. If both $\mathcal{F}_{1}$ and $\mathcal{F}_{m N_{0}}$ are tight frames of $L^{2}$, then for any integer $s,\left\{M^{j / 2} \psi_{l}\left(M^{j} \cdot-k / N_{0}-s /\left(m N_{0}\right)\right): j, k \in\right.$ $\mathbf{Z}, l=1, \ldots, L\}$ is also a tight frame of $L^{2}$. 
Note that for the case where $b^{-1} \in \mathbf{Z}$ is a factor of $n_{a}$, condition (10) is equivalent to

$$
\begin{aligned}
& \sum_{l=1}^{L} \sum_{j=1}^{\infty} \overline{\widehat{\psi}_{l}\left(n_{a}^{j} \omega\right)} \widehat{\psi}_{l}\left(n_{a}^{j}(\omega+2 \pi d)\right) \\
& \quad+\sum_{l=1}^{L} \overline{\widehat{\psi}_{l}(\omega)} \widehat{\psi}_{l}(\omega+2 \pi d) \delta_{d, b^{-1} \mathbf{z}}=0 \quad \text { a.e. } \omega \in \mathbf{R}
\end{aligned}
$$

for all $d \in \mathbf{Z} \backslash n_{a} \mathbf{Z}$, where we set $\delta_{d, b^{-1} \mathbf{Z}}=1$ for $d \in b^{-1} \mathbf{Z}$ and $\delta_{d, b^{-1} \mathbf{Z}}=0$ otherwise. Then by (18), Theorem A, and Theorem 1, we have the following result about preservation of tightness for oversampling affine frames with dilation $a$ that satisfies (8) and with $m$ being a factor of $n_{a}$.

Theorem 3. Let $a>1$ satisfy (8), $m \mid n_{a}$, and assume that $\left\{a^{j / 2} \psi_{l}\left(a^{j} \cdot-k\right): j, k \in\right.$ $\mathbf{Z}, l=1, \ldots, L\}$ is a tight frame of $L^{2}$. Set $\Phi=\left[\begin{array}{lll}\psi_{1} & \ldots & \psi_{L}\end{array}\right]^{T}$. Then the following statements are equivalent:

(i) $\left\{a^{j / 2} \psi_{l}\left(a^{j} \cdot-k / m\right): j, k \in \mathbf{Z}, l=1, \ldots, L\right\}$ is a tight frame of $L^{2}$;

$$
\sum_{l=1}^{L} \overline{\widehat{\psi}_{l}(\omega)} \widehat{\psi}_{l}(\omega+2 \pi d)=0 \quad \text { a.e. } \omega \in \mathbf{R}
$$

for all $d \in \mathbf{Z} \backslash m \mathbf{Z}$;

(iii) $Q_{0}$ is shift-invariant with respect to $\frac{1}{m} \mathbf{Z}$;

(iv) $Q_{0, m}=m Q_{0}$;

(v) there exists some $2 \pi$-periodic unitary matrix $A(\omega)$ of dimension $L$ such that

$$
e^{-i \omega / m} \widehat{\Phi}(\omega)=A(\omega) \widehat{\Phi}(\omega) \quad \text { a.e. } \omega \in \mathbf{R} .
$$

For the case $b^{-1}=n_{a}^{\gamma} m \in \mathbf{Z}$ with $0 \leq \gamma \in \mathbf{Z}$ and $m \mid n_{a}$, it is easy to check that condition (10) is equivalent to

$$
\begin{aligned}
& \sum_{l=1}^{L} \sum_{j=\gamma+1}^{\infty} \overline{\widehat{\psi}_{l}\left(n_{a}^{j} \omega\right)} \widehat{\psi}_{l}\left(n_{a}^{j}(\omega+2 \pi d)\right) \\
& \quad+\sum_{l=1}^{L} \overline{\widehat{\psi}_{l}\left(n_{a}^{\gamma} \omega\right)} \widehat{\psi}_{l}\left(n_{a}^{\gamma}(\omega+2 \pi d)\right) \delta_{d, m \mathbf{Z}}=0 \quad \text { a.e. }
\end{aligned}
$$

for all $d \in \mathbf{Z} \backslash n_{a} \mathbf{Z}$. This together with (7) give the following extension of the equivalence of (i) and (iv) in Theorem 1.

Theorem 4. Let $2 \leq M \in \mathbf{Z}, 0 \leq \gamma \in \mathbf{Z}, 2 \leq m \leq M$ with $\operatorname{gcd}(m, M)=m$, and assume that $\Psi=\left\{\psi_{1}, \ldots, \psi_{L}\right\} \subset L^{2}$ generates a tight affine frame $\mathcal{F}_{1}$ in (1) of $L^{2}$. Then $\mathcal{F}_{M^{\gamma} m}$ in (11) is a tight frame of $L^{2}$ if and only if

$$
\begin{aligned}
Q_{0, M^{\gamma} m}= & M^{\gamma} m D_{\gamma} Q_{0} D_{-\gamma} \\
& +m \sum_{j=0}^{\gamma-1} M^{j}\left(D_{j} Q_{0, M^{\gamma-j}} D_{-j}-D_{j+1} Q_{0, M^{\gamma-j}} D_{-j-1}\right) .
\end{aligned}
$$




\section{Preliminary Results}

Given a measurable set $E$, we let $M\left(E, \ell_{0}\right)$ be the space of all sequences $\left\{\alpha_{n}(\omega)\right\}_{n=-\infty}^{\infty}$ of measurable functions on $E$, such that for almost all $\omega \in E$, $\alpha_{n}(\omega)=0$ for all but finitely many $n \in \mathbf{Z}$, i.e.,

$$
\bigcup_{N \geq 1}\left(\bigcap_{|n| \geq N}\left\{\omega \in E: \alpha_{n}(\omega)=0\right\}\right)=E .
$$

Here, we say that two measurable sets $A$ and $B$ are equal, denoted by $A=B$, if both $A \backslash B$ and $B \backslash A$ have zero Lebesgue measure. For a sequence $X=\left\{x_{n}(\omega) \in\right.$ $\left.\mathbf{C}^{L}\right\}_{n=-\infty}^{\infty}$ of vector-valued measurable functions on a measurable set $E$, let

$$
S(E, X):=\left\{\sum_{n=-\infty}^{\infty} \alpha_{n}(\omega) x_{n}(\omega):\left\{\alpha_{n}(\omega)\right\}_{n=-\infty}^{\infty} \in M\left(E, \ell_{0}\right)\right\} .
$$

We remark that $S(E, X)$ is well defined since the summation for $n$ in the definition is taken over a finite set for almost all $\omega \in E$.

For the proof of Theorem 1, we need the following technical lemma.

Lemma 1. Let $1 \leq L \in \mathbf{Z}$, let $E$ be a measurable set, and let $X=\left\{x_{n}(\omega)\right\}_{n=-\infty}^{\infty}$ be a sequence of vector-valued measurable functions $x_{n}(\omega) \in \mathbf{C}^{L}, n \in \mathbf{Z}$, on $E$. Then there exists an $L$-dimensional square matrix $P(\omega)$ of measurable functions on E such that

and

$$
\begin{gathered}
P(\omega) v \in S(E, X) \quad \text { for all } v \in \mathbf{C}^{L}, \\
\overline{P(\omega)^{T}}=P(\omega), P(\omega)^{2}=P(\omega) \quad \text { a.e. } \omega \in E,
\end{gathered}
$$

In order to prove Lemma 1, we need the following result.

Lemma 2. Let $Y=\left\{y_{n}(\omega)\right\}_{n=-\infty}^{\infty}$ and $Z=\left\{z_{n}(\omega)\right\}_{n=-\infty}^{\infty}$ be sequences of vectorvalued measurable functions on a measurable set $E$. If $z_{n}(\omega) \in S(E, Y)$ for all $n \in \mathbf{Z}$, then $S(E, Z) \subset S(E, Y)$.

Proof. Write

$$
z_{n}(\omega)=\sum_{k=-\infty}^{\infty} \beta_{n, k}(\omega) y_{k}(\omega)
$$

with $\left\{\beta_{n, k}(\omega)\right\}_{k=-\infty}^{\infty} \in M\left(E, \ell_{0}\right)$ for all $n \in \mathbf{Z}$. Then for any sequence $\left\{\alpha_{n}(\omega)\right\}_{n=-\infty}^{\infty}$ of measurable functions in $M\left(E, \ell_{0}\right)$, we have

$$
\sum_{n=-\infty}^{\infty} \alpha_{n}(\omega) z_{n}(\omega)=\sum_{k=-\infty}^{\infty} \gamma_{k}(\omega) y_{k}(\omega)
$$

where $\gamma_{k}(\omega)=\sum_{n=-\infty}^{\infty} \alpha_{n}(\omega) \beta_{n, k}(\omega)$. Therefore, it suffices to show that

$$
\left\{\gamma_{k}(\omega)\right\}_{k=-\infty}^{\infty} \in M\left(E, \ell_{0}\right) \text {. }
$$

For any $0<C \in \mathbf{R}, n \in \mathbf{Z}$, and $1 \leq N, K \in \mathbf{Z}$, set

$$
\begin{array}{lll}
E_{C, K}:=\{\omega \in E:|\omega| \leq C & \text { and } \quad \gamma_{k}(\omega)=0 & \text { for all }|k| \geq K\} \\
F_{C, N}:=\{\omega \in E:|\omega| \leq C & \text { and } \quad \alpha_{n}(\omega)=0 & \text { for all }|n| \geq N\}
\end{array}
$$


and

$$
G_{C, n, K}:=\left\{\omega \in E:|\omega| \leq C \quad \text { and } \quad \beta_{n, k}(\omega)=0 \quad \text { for all }|k| \geq K\right\} .
$$

It is easy to see that for any $1 \leq K, N \in \mathbf{Z}$ and $0<C \in \mathbf{R}$,

$$
F_{C, N} \cap\left(\bigcap_{|n| \leq N-1} G_{C, n, K}\right) \subset E_{C, K} .
$$

By the definition of $M\left(E, \ell_{0}\right)$, the proof of (24) reduces to the existence of an integer $1 \leq K_{1}=K_{1}(\epsilon, C)$ for any pre-assigned positive constants $\epsilon$ and $C$, such that

$$
\operatorname{meas}\left((E \cap\{\omega:|\omega| \leq C\}) \backslash E_{C, K_{1}}\right)<\epsilon,
$$

where meas $(A)$ denotes the Lebesgue measure of a measurable set $A$. Since

$$
\left\{\alpha_{n}(\omega)\right\}_{n=-\infty}^{\infty} \in M\left(E, \ell_{0}\right),
$$

there exists an integer $N_{1} \geq 1$ such that

$$
\operatorname{meas}\left((E \cap\{\omega:|\omega| \leq C\}) \backslash F_{C, N_{1}}\right)<\epsilon / 3 .
$$

From the assumption $\left\{\beta_{n, k}(\omega)\right\}_{k=-\infty}^{\infty} \in M\left(E, \ell_{0}\right)$ for any $n \in \mathbf{Z}$, we may find an integer $K_{1} \geq 1$ so that

$$
\operatorname{meas}\left((E \cap\{\omega:|\omega| \leq C\}) \backslash G_{C, n, K_{1}}\right)<\epsilon /\left(3 N_{1}\right)
$$

for all $n \in \mathbf{Z}$ with $|n| \leq N_{1}-1$. Hence, (26) follows from (25), (27), and (28).

Proof of Lemma 1. Set $x_{0, n}(\omega)=x_{n}(\omega), n \in \mathbf{Z}$, and $X_{0}=X$, and define inductively, $e_{l}(\omega)$ and $X_{l}=\left\{x_{l, n}(\omega)\right\}_{n=-\infty}^{\infty}, 1 \leq l \leq L$, by

$$
e_{l}(\omega)= \begin{cases}x_{l-1, I\left(X_{l-1}\right)(\omega)}(\omega) /\left|x_{l-1, I\left(X_{l-1}\right)(\omega)}(\omega)\right| & \text { if } \quad \omega \in E \backslash E_{l-1}, \\ 0 & \text { if } \omega \in E_{l-1},\end{cases}
$$

and

$$
x_{l, n}(\omega)=x_{l-1, n}(\omega)-e_{l}(\omega){\overline{e_{l}(\omega)}}^{T} x_{l-1, n}(\omega), n \in \mathbf{Z},
$$

where

$$
E_{l-1}=\left\{\omega \in E: x_{l-1, n}(\omega)=0 \text { for all } n \in \mathbf{Z}\right\},
$$

and where $I\left(X_{l-1}\right)(\omega)$ is an integer-valued measurable function of $\omega \in E$, so chosen that

$$
x_{l-1, I\left(X_{l-1}\right)(\omega)}(\omega) \neq 0 \quad \text { for any } \quad \omega \in E \backslash E_{l-1} .
$$

For instance, given a sequence $Y=\left\{y_{n}(\omega)\right\}_{-\infty}^{\infty}$ of measurable functions, for any $\omega \in \bigcup_{n=-\infty}^{\infty} \operatorname{supp} y_{n}$, we may choose $I(Y)(\omega)$ to be the smallest integer $n$ such that $y_{n}(\omega) \neq 0$ and $\left|y_{n^{\prime}}(\omega)\right|=0$ for all $n^{\prime} \in \mathbf{Z}$ with $\left|n^{\prime}\right|<|n|$.

Now by (29), (30), and (31), we see that for any $1 \leq l \leq L$ and $n \in \mathbf{Z}, e_{l}(\omega)$ and $x_{l, n}(\omega)$ are measurable functions on $E, e_{l}(\omega), x_{l, n}(\omega) \in S\left(E, X_{l-1}\right)$, and 
${\overline{x_{l, n}(\omega)}}^{T} e_{l}(\omega)=0$. This together with Lemma 2 leads to

$$
S\left(E, X_{L}\right) \subset S\left(E, X_{L-1}\right) \subset \ldots \subset S\left(E, X_{1}\right) \subset S(E, X),
$$

and for all $1 \leq l \leq L$,

$$
e_{l}(\omega) \in S(E, X)
$$

and

$$
\overline{y(\omega)}^{T} e_{l}(\omega)=0 \text { for all } y(\omega) \in S\left(E, X_{l}\right) .
$$

By (29), (32), (33), and (35), we obtain

$$
{\overline{e_{l}(\omega)}}^{T} e_{l^{\prime}}(\omega)= \begin{cases}\chi_{E \backslash E_{l-1}}(\omega) & \text { if } l=l^{\prime} \\ 0 & \text { if } l \neq l^{\prime}\end{cases}
$$

Hence from (34) and (36), we see that the choice of

$$
P(\omega)=\sum_{l=1}^{L} e_{l}(\omega){\overline{e_{l}(\omega)}}^{T}
$$

gives (21) and (22). To verify (23), we set

$$
E_{L}=\left\{\omega \in E: x_{L, n}(\omega)=0 \text { for all } n \in \mathbf{Z}\right\},
$$

and observe that this set contains the sets introduced in (31), namely,

$$
E_{0} \subset E_{1} \subset \cdots \subset E_{L-1} \subset E_{L} .
$$

Therefore by (36), we have

$$
\overline{Q(\omega)}^{T} Q(\omega)=I_{L} \quad \text { a.e. } \omega \in E \backslash E_{L},
$$

where the $L$-dimensional square matrix $Q(\omega)$ of measurable functions is defined by $Q(\omega)=\left[e_{1}(\omega) \ldots e_{L}(\omega)\right]$. It is easy to see that $P(\omega)=Q(\omega) \overline{Q(\omega)}^{T}$ which, together with (37), leads to

$$
P(\omega)=I_{L} \quad \text { a.e. } \omega \in E \backslash E_{L} .
$$

By (301) and (36), we obtain

$$
\begin{aligned}
x_{L, n}(\omega) & =x_{n}(\omega)-\sum_{l=1}^{L} e_{l}(\omega){\overline{e_{l}(\omega)}}^{T} x_{l-1, n}(\omega) \\
& =x_{n}(\omega)-P(\omega) x_{n}(\omega) \quad \text { a.e. } \omega \in E .
\end{aligned}
$$

Thus, combining (38) and (39), we have

$$
x_{L, n}(\omega) \equiv 0 \quad \text { a.e. } \omega \in E \backslash E_{L} \quad \text { for all } n \in \mathbf{Z} .
$$

This, together with (39) and the definition of the set $E_{L}$, imply (23). 


\section{Proof of the MAIN RESUlts}

We only give the proof of Theorem 1, since the other three theorems and Corollary 1 follow accordingly. We divide the proof of Theorem 1 into the following steps: (i) $\Longrightarrow$ (ii) $\Longrightarrow($ iii) $\Longrightarrow$ (iv) $\Longrightarrow(\mathrm{i})$, and (v) $\Longrightarrow$ (ii) $\Longrightarrow(\mathrm{v})$. The proof of (ii) $\Longrightarrow(\mathrm{v})$ is the most technical part in our proof, and will be dealt with last.

Set $\mathcal{G}=\left\{\psi_{l}(\cdot-n / m): 1 \leq l \leq L, 0 \leq n \leq m-1\right\}$. Then for $\mathcal{F}_{m}$ in (11), we have

$$
\mathcal{F}_{m}=\left\{M^{j / 2} g\left(M^{j} \cdot-k\right): g \in \mathcal{G}, j, k \in \mathbf{Z}\right\} .
$$

By direct computation, we obtain

$$
\sum_{g \in \mathcal{G}} \sum_{j \in \mathbf{Z}}\left|\widehat{g}\left(M^{j} \omega\right)\right|^{2}=m \sum_{l=1}^{L} \sum_{j \in \mathbf{Z}}\left|\widehat{\psi}_{l}\left(M^{j} \omega\right)\right|^{2},
$$

and for all integers $d$,

$$
\begin{aligned}
& \sum_{g \in \mathcal{G}} \sum_{j=0}^{\infty} \overline{\widehat{g}\left(M^{j} \omega\right)} \widehat{g}\left(M^{j}(\omega+2 \pi d)\right) \\
= & \sum_{n=0}^{m-1} \sum_{l=1}^{L} \sum_{j=0}^{\infty} \overline{\widehat{\psi}_{l}\left(M^{j} \omega\right)} \widehat{\psi}_{l}\left(M^{j}(\omega+2 \pi d)\right) e^{i 2 \pi d n M^{j} / m} \\
= & m \sum_{l=1}^{L} \sum_{j=1}^{\infty} \overline{\widehat{\psi}_{l}\left(M^{j} \omega\right)} \widehat{\psi}_{l}\left(M^{j}(\omega+2 \pi d)\right)+m \sum_{l=1}^{L} \overline{\widehat{\psi}_{l}(\omega)} \widehat{\psi}_{l}(\omega+2 \pi d) \delta_{d, m \mathbf{Z}} .
\end{aligned}
$$

Therefore, by (40), (41), the assumption (i), and Theorem A, we have

$$
\sum_{l=1}^{L} \sum_{j=1}^{\infty} \overline{\widehat{\psi}_{l}\left(M^{j} \omega\right)} \widehat{\psi}_{l}\left(M^{j}(\omega+2 \pi d)\right)+\sum_{l=1}^{L} \overline{\widehat{\psi}_{l}(\omega)} \widehat{\psi}_{l}(\omega+2 \pi d) \delta_{d, m \mathbf{Z}}=0
$$

for any $d \in \mathbf{Z} \backslash M \mathbf{Z}$. Similarly by Theorem A, (40) and (41) with $m=1$, and the assumption that $\mathcal{F}_{1}$ is a tight frame of $L^{2}$, we also have

$$
\sum_{l=1}^{L} \sum_{j=0}^{\infty} \overline{\widehat{\psi}_{l}\left(M^{j} \omega\right)} \widehat{\psi}_{l}\left(M^{j}(\omega+2 \pi d)\right)=0
$$

for any $d \in \mathbf{Z} \backslash M \mathbf{Z}$. Hence (ii) follows from (42) and (43).

Taking the Fourier transform on both sides of (4) leads to

$$
\left(Q_{0} f\right)^{\wedge}(\omega)=\sum_{l=1}^{L} \sum_{k \in \mathbf{Z}} \widehat{f}(\omega+2 k \pi) \overline{\widehat{\psi}_{l}(\omega+2 k \pi)} \widehat{\psi}_{l}(\omega), j \geq 0 .
$$

Hence, for any $f \in L^{2}$ and $d \in \mathbf{Z}$,

$$
\begin{aligned}
& \left(\tau_{d / m} Q_{0} \tau_{-d / m} f-Q_{0} f\right)^{\wedge}(\omega) \\
= & \sum_{l=1}^{L} \sum_{k \in \mathbf{Z}} \widehat{f}(\omega+2 k \pi) \overline{\widehat{\psi}_{l}(\omega+2 k \pi)} \widehat{\psi}_{l}(\omega)\left(e^{2 i d k \pi / m}-1\right) .
\end{aligned}
$$

This, together with assumption (ii), lead to the shift-invariance of the operator $Q_{0}$ with respect to $\frac{1}{m} \mathbf{Z}$, and this establishes (ii) $\Longrightarrow$ (iii). 
To prove (iii) $\Longrightarrow$ (iv), note that

$$
Q_{0, m}=\sum_{k=0}^{m-1} \tau_{k / m} Q_{0} \tau_{-k / m}
$$

from its definition (4). Hence (iv) follows from (14) and (44).

To prove (iv) $\Longrightarrow(\mathrm{i})$, note that since $\mathcal{F}_{1}$ is a tight frame, the function $H$ in (3) is bounded. Therefore by (7), $\mathcal{F}_{m}$ in (11) is a tight frame if and only if

$$
\sum_{j \in \mathbf{Z}} D_{-j} Q_{0, m} D_{j}=A I
$$

for some positive constant $A$. Hence (i) follows from (45), the tight frame assumption on $\mathcal{F}_{1}$, and the hypothesis $Q_{0, m}=m Q_{0}$.

Let (v) be satisfied. Then we have

$$
e^{i(\omega+2 \pi d) / m} \widehat{\Phi}(\omega+2 d \pi)=A(\omega) \widehat{\Phi}(\omega+2 d \pi)
$$

for any integer $d$. Thus,

$$
\begin{aligned}
e^{2 d i \pi / m} \overline{\widehat{\Phi}(\omega)^{T}} \widehat{\Phi}(\omega+2 \pi d) & =\overline{\widehat{\Phi}(\omega)^{T}} \overline{A(\omega)^{T}} A(\omega) \widehat{\Phi}(\omega+2 \pi d) \\
& =\overline{\Phi(\omega)^{T}} \widehat{\Phi}(\omega+2 \pi d)
\end{aligned}
$$

which implies (ii).

Finally, we come to the proof of (ii) $\Longrightarrow(\mathrm{v})$. Let $P(\omega)$ be the $L$-dimensional square matrix of measurable functions on $[-m \pi, m \pi)$ in Lemma 1, with $E=[-m \pi, m \pi)$ and $X=\{\widehat{\Phi}(\omega+2 m n \pi)\}_{n=-\infty}^{\infty}$. For notational convenience, we denote the $2 m \pi$ periodization of $P(\omega)$ again by $P(\omega)$. Then by Lemma 1, we have

$$
P(\omega+2 m \pi)=P(\omega), \overline{P(\omega)^{T}}=P(\omega), P(\omega)^{2}=P(\omega) \text { a.e. } \omega \in \mathbf{R},
$$

and

$$
\widehat{\Phi}(\omega)=P(\omega) \widehat{\Phi}(\omega) \quad \text { a.e. } \omega \in \mathbf{R},
$$

where $\mathcal{G}_{m}=\{\widehat{\Phi}(\omega+2 m n \pi)\}_{n=-\infty}^{\infty}$. By (46) and assumption (ii), we obtain

$$
\overline{P(\omega)^{T}} P(\omega+2 d \pi)=0 \quad \text { for all } d \in \mathbf{Z} \backslash m \mathbf{Z} .
$$

Therefore, the function

$$
A(\omega):=I_{L}+\sum_{d=0}^{m-1}\left(e^{i(\omega+2 \pi d) / m}-1\right) P(\omega+2 \pi d)
$$


is an $L$-dimensional square matrix of measurable functions that satisfies $A(\omega+2 \pi)$ $=A(\omega)$ by (47), and

$$
\begin{aligned}
& \overline{A(\omega)^{T}} A(\omega) \\
= & I_{L}+\sum_{d=0}^{m-1}\left(e^{i(\omega+2 \pi d) / m}-1\right) P(\omega+2 \pi d)+\sum_{d=0}^{m-1}\left(e^{-i(\omega+2 \pi d) / m}-1\right) \overline{P(\omega+2 \pi d)^{T}} \\
& +\left(\sum_{d=0}^{m-1}\left(e^{-i(\omega+2 \pi d) / m}-1\right) \overline{P(\omega+2 \pi d)^{T}}\right) \\
& \times\left(\sum_{d=0}^{m-1}\left(e^{i(\omega+2 \pi d) / m}-1\right) P(\omega+2 \pi d)\right) \\
= & I_{L}+\sum_{d=0}^{m-1}\left(e^{i(\omega+2 \pi d) / m}-1\right) P(\omega+2 \pi d)+\sum_{d=0}^{m-1}\left(e^{-i(\omega+2 \pi d) / m}-1\right) P(\omega+2 \pi d) \\
& +\sum_{d=0}^{m-1}\left(e^{-i(\omega+2 \pi d) / m}-1\right) \times\left(e^{i(\omega+2 \pi d) / m}-1\right) P(\omega+2 d \pi) \\
= & I_{L},
\end{aligned}
$$

where we have used (47) and (49) to obtain the second equality. Moreover, by (47), (48), and (49), we get

$$
\begin{aligned}
A(\omega) \widehat{\Phi}(\omega) & =\widehat{\Phi}(\omega)+\sum_{d=0}^{m-1}\left(e^{i(\omega+2 \pi d) / m}-1\right) P(\omega+2 \pi d) \widehat{\Phi}(\omega) \\
& =\widehat{\Phi}(\omega)+\sum_{d=0}^{m-1}\left(e^{i(\omega+2 \pi d) / m}-1\right) \overline{P(\omega+2 \pi d)^{T}} P(\omega) \widehat{\Phi}(\omega) \\
& =e^{i \omega / m} \widehat{\Phi}(\omega) .
\end{aligned}
$$

This completes the proof of (v), and hence Theorem 1.

\section{ACKNOWLEDGMENT}

This research was completed while the second author was visiting the Institute of Computational Harmonic Analysis (ICHA) of the University of Missouri-St. Louis during November and December, 2000. The kind hospitality extended to him by the Department of Mathematics, and particularly Kai Bittner, Wenjie He, and Joachim Stoeckler of ICHA, is greatly appreciated.

\section{REFERENCES}

1. A. Aldroubi, Portraits of frames, Proc. Amer. Math. Soc. 123 (1995), 1661-1668. MR 95g:46037

2. A. Aldroubi, Q. Sun and W.-S. Tang, p-frames and shift invariant subspaces of $L^{p}$, J. Fourier Anal. Appl. 7 (2001), 1-21. MR 2002c: 42046

3. M. Bownik, A characterization of affine dual frames in $L^{2}\left(\mathbf{R}^{n}\right)$, Appl. Comp. Harmonic Anal. 8 (2000), 203-221. MR 2001d:42019

4. A. Calogero, Wavelets on general lattices, ERA Amer. Math. Soc. 5 (1999), 1-10. MR 99i: 42042

5. A. Calogero, A characterization of wavelets on general lattices, J. Geom. Anal. 10 (2000), 597-622. MR 2002b:42051 
6. C. K. Chui, W. Czaja, M. Maggioni and G. Weiss, Characterization of general tight wavelet frames with matrix dilations and tightness preserving oversampling, J. Fourier Anal. Appl., To appear.

7. C. K. Chui and X. L. Shi, Bessel sequences and affine frames, Appl. Comp. Harmonic Anal. 1 (1993), 29-49. MR 95b:42028

8. C. K. Chui and X. L. Shi, $n \times$ oversampling preserves any tight affine frame for odd $n$, Proc. Amer. Math. Soc. 121 (1994), 511-517. MR 94h:42052

9. C. K. Chui and X. L. Shi, Inequalities on matrix-dilated Littlewood-Paley energy functions and oversampled affine frames, SIAM J. Math. Anal. 28 (1997), 213-232. MR 98b:42046

10. C. K. Chui and X. L. Shi, Orthonormal wavelets and tight frames with arbitrary real dilations, Appl. Comp. Harmonic Anal. 9 (2000), 243-264. MR 2002a:42025

11. R. G. Catalán, Oversampling and preservation of tightness in affine frames, Proc. Amer. Math. Soc. 130 (2002), 1031-1034.

12. E. Hernandez and G. Weiss, A First Course on Wavelets, CRC Press, Boca Raton, FL, 1996. MR 97i: 42015

13. J. Stoeckler, "Multivariate Affine Frames", Habil.-Schr. Thesis, Duisburg Univ., 1996.

14. E. Weber, On the translation invariance of wavelet subspaces, J. Fourier Anal. Appl. 6 (2000), 551-558. MR 2001h:42057

Department of Mathematics and Computer Science, University of Missouri-St. Louis, St. Louis, Missouri 63121-4499 - and - Department of Statistics, Stanford University, Stanford, CALIFornia 94305

E-mail address: cchui@stat.stanford.edu

Department of Mathematics, National University of Singapore, Singapore 119260 , REPUBlic OF Singapore

E-mail address: matsunqy@nus.edu.sg 